\title{
Conselho Editorial ad hoc
}

Agradecemos aos avaliadores que contribuíram para assegurar o padrão de qualidade da revista Ensaio: Avaliação e Políticas Públicas em Educação.

Abelardo Bento Araújo (Centro Federal de Educação Tecnológica de Minas Gerais, CEFET-MG, Belo Horizonte, MG, Brasil); Adelia Maria Miglievich Ribeiro (Universidade Federal do Espírito Santo - UFES, Vitória, ES, Brasil); Adolfo Ignácio Calderón (Pontifícia Universidade Católica de Campinas PUC-Campinas, Campinas, SP, Brasil); Adriana Barroso de Azevedo (Universidade Metodista de São Paulo - UMESP, São Paulo, SP, Brasil); Adriana Bauer (Fundação Carlos Chagas e USP, São Paulo, SP, Brasil); Adriana Rivoire Menelli de Oliveira (Centro Universitário Metodista, Porto Alegre, RS, Brasil); Alba Garcia-Barrera (Universidad a Distancia de Madrid - Udima, Madrid, España); Aílton Paulo de Oliveira Júnior (Universidade de São Paulo - USP, São Paulo, SP, Brasil); Aline Battisti Archer (Universidade Federal de Santa Catarina, UFSC, Florianópolis, SC, Brasil); Alexandre Pereira Salgado Junior (USP, Universidade de São Paulo, São Paulo, SP,Brasil); Ana Paula Serrata Malfitano (Universidade Federal de São Carlos, UFSCar, São Carlos, SP, Brasil);Ana Rita Faria (Instituto de Educação da Universidade de Lisboa, Lisboa, Portugal); Andrea Tereza Brito Ferreira (Universidade Federal de Pernambuco - UFPE, Recife, PE, Brasil); Amos Nascimento (University of Washington, Washington, United States of America); Ana Clara Bortoleto Nery (Universidade Estadual Paulista Júlio de Mesquita Filho UNESP, São Paulo, SP, Brasil); Ana Cristina Prado de Oliveira (UNIRIO Universidade Federal do Estado do Rio de Janeiro, UFRJ, Rio de Janeiro, RJ, Brasil); Ana Maria Eyng (Pontifícia Universidade Católica do Paraná PUC-PR, Curitiba, PR, Brasil); Andréa Barbosa Gouveia (Universidade Federal do Paraná - UFPR, Curitiba, PR, Brasil); Andres Chiappe (Universidad de La Sabana, Chía, Cundinamarca, Colômbia); Ângelo Ricardo de Souza (Universidade Federal do Paraná - UFPR, Curitiba, PR, Brasil); Annie Gomes Redig (Universidade Estadual do Rio de Janeiro - UERJ, Rio de Janeiro, RJ, Brasil); Angela Mágda Rodrigues Virgolim (Universidade de Brasília - UnB, Brasília, DF, Brasil); Ângela Maria Cristina Uchoa de Abreu Branco (Universidade de Brasília - UnB, Brasília, DF, Brasil); Ângela Rabelo Barreto (Universidade de Brasília - UnB, Brasília, DF, Brasil); Antonio Luzón 
(Universidad de Granada, Granada, España); António Manuel Duarte (Universidade de Lisboa- Ulisboa, Lisboa, Portugal); Armando Terribili Filho (Fundação Armando Álvares Penteado - FAAP, São Paulo, SP, Brasil); Artur Marecos Parreira e Moreira Gonçalves (Universidade Lusófona, Lisboa, Portugal); Bertha de Borja Reis do Vale (Universidade do Estado do Rio de Janeiro - UERJ, Rio de Janeiro, RJ, Brasil); Carmen Rodríguez-Martínez (Universidad de Málaga, Málaga, España); Carolina Iturra Chile (Universidad de Talca, Talca, Chile); Cintia Schwamberger (Universidad Nacional de San Martín, Buenos Aires, Argntina); Claudia Daniele Leite (Instituto Federal de Educação, Ciência e Tecnologia de Pernambuco - IFPE, Recife, PE, Brasil); Cristina Costa Lobo (Universidade Portucalense Infante D Henrique, Porto, Portugal); Cyntia Paes de Carvalho (Pontifícia Universidade Católica do Rio de Janeiro - PUC-Rio, Rio de Janeiro, RJ, Brasil); Dalton Francisco de Andrade (Universidade Federal de Santa Catarina - UFSC, Florianópolis, SC, Brasil); Denize Sepulveda (Universidade do Estado do Rio de Janeiro-UERJ, Rio de Janeiro, RJ, Brasil); Diana da Veiga Manderlet (Universidade do Estado do Rio de Janeiro-UERJ, Rio de Janeiro - UERJ, Rio de Janeiro, RJ, Brasil); Dinorá Zucchetti (Universidade Federal do Rio Grande do Sul - UFRGS, Porto Alegre, RS, Brasil); Domingos Manuel de Barros Fernandes (Universidade de Lisboa, Lisboa Portugal); Douglas da Silva Tinti (Universidade Cidade de São Paulo - UNICID, São Paulo, SP, Brasil); Edicléa Mascarenhas Fernandes (Universidade do Estado do Rio de Janeiro- UERJ, Rio de Janeiro, RJ, Brasil); Edson Maciel Peixoto (Instituto Federal de Educação, Ciência e Tecnologia do Espírito Santo-IFES, Cachoeiro de Itapemirim, ES, Brasil); Edméa Oliveira dos Santos (Universidade do Estado do Rio de Janeiro- UERJ, Rio de Janeiro, RJ, Brasil); Eimard Gomes Antunes do Nascimento (Universidade de Aveiro - UA, Aveiro, Portugal); Eliana Borges Correia de Albuquerque (Universidade Federal de Pernambuco - UFPE, Recife, PE, Brasil); Eliana de Moura (Pontifícia Universidade Católica do Rio Grande do Sul - PUCRS, Porto Alegre, RS, Brasil); Elisângela Bernado (Universidade Federal do Estado do Rio de Janeiro - UNIRIO, Rio de Janeiro, RJ, Brasil); Elsio José Corá (Universidade Federal da Fronteira Sul - UFFS, Chapecó, SC); Elton Luiz Nardi (Universidade do Oeste de Santa Catarina- Unoesc, Joaçaba, SC, Brasil); Emilia Maria da Trindade Prestes (Universidade Federal da Paraíba-UFPB, João Pessoa, PB, Brasil); Erinaldo Ferreira do Carmo (Universidade Federal de Pernambuco - UFPE, Recife, Pernambuco, Brasil); Fátima Aparecida da Silva Dias (Universidade Norte do Pará - Unopar, Londrina, PR, Brasil); Fátima Bayma de Oliveira (Fundação Getúlio Vargas - FGV, Rio de Janeiro, RJ, Brasil); Fátima Maria Neves (Universidade Estadual de Maringá - UEM, Maringá, Paraná, Brasil); Francisco José da Silveira Lobo Neto (Fundação Oswaldo Cruz - Fiocruz, Rio de Janeiro, RJ, Brasil); Francisco Remolina- 
Caviedes (Universidad Industrial de Santander - UIS, Bucaramanga, Santander, Colômbia); Geraldo Caliman (Universidade Católica de Brasília - UCB, Brasília, DF, Brasil); Giselle Cristina Martins Real (Universidade Federal da Grande Dourados - UFGD, Dourados, MS, Brasil); Gonzalo Donoso (Universidad de Chile, Santiago, Chile); Guilherme Issamu Hirata (IDados, Rio de Janeiro, RJ, Brasil); Gustavo Fontoura de Souza (Instituto Federal de Educação, Ciência e Tecnologia do Rio Grande do Norte - IFRN, Natal, RN, Brasil); Gustavo Marchisotti (Empresa de Tecnologia e Informações da Previdência, Dataprev, Rio de Janeiro, RJ, Brasil); Helder Nuno Ricardo Ferraz (Universidade do Porto, FPCEUP, Porto, Portugal); Ilma Ferreira Machado (Universidade do Estado de Mato Grosso - UNEMAT, Cáceres, MT, Brasil); Irene Cadime (Universidade do Minho - UMinho, Braga, Portugal); Ivanete Bellucci Almeida (Faculdade de Tecnologia Victor Civita - Fatec Tatuape - CEETPS, São Paulo, SP, Brasil); Ivár César Oliveira de Vasconcelos (Universidade Paulista - UNIP, Brasília, DF, Brasil); Jacira da Silva Câmara (Universidade Católica de Brasília - UCB, Brasília, DF, Brasil); Jaime Farias Dresch (Universidade do Planalto Catarinense - UNIPLAC, Lages, SC, Brasil); Janaina Specht da Silva Menezes (Universidade Federal do Rio de Janeiro - UFRJ, Rio de Janeiro, RJ, Brasil); Janete Palazzo (Universidade Católica de Brasília - UCB, Brasília, DF, Brasil); Javier Coollado-Ruano (Universidad Nacional de Educación - UNAE , Cañar, Ecuador); Javier Pericacho Goméz (Universidad Antonio de Nebrija, Madrid, España); Jefferson Mainardes (Universidade Estadual de Ponta Grossa-UEPG, Ponta Grossa, PR, Brasil); Jevuks Matheus de Araújo (Universidade Federal da Paraíba - UFPB, João Pessoa, PB, Brasil); João Casqueira Cardoso (Universidade Fernando Pessoa, Porto, Portugal); João dos Reis Silva Junior (Universidade Federal de São Carlos - UFSCar, São Carlos, SP, Brasil); Jomária Mata de Lima Alloufa (Universidade Federal do Rio Grande do Norte - UFRN, Natal, RN, Brasil); Jorge Luis Yangali (Universidad Nacional del Centro del Perú, Huancayo, Perú); Juan Carlos Castellanos Ramírez (Universidad Autónoma de Baja California, Mexicali, México); Julio Cesar Godoy Bertolin (Universidade de Passo Fundo - UPF, Passo Fundo, RS, Brasil); Jussara Cristina Barboza Tortela Pontifícia Universidade Católica de Campinas (PUC-Campinas, Campinas, SP, Brasil); Laelia Portela Moreira (Universidade Estácio de Sá - UNESA, Rio de Janeiro, RJ, Brasil); Leandro Marino (Fundação Cesgranrio, Rio de Janeiro, RJ, Brasil); Ligia Leite Silva (Fundação Cesgranrio, Rio de Janeiro, RJ, Brasil); Lincoln de Araújo Santos (Universidade do Estado do Rio de Janeiro- UERJ, Rio de Janeiro, RJ, Brasil); Lincoln Tavares Silva (Universidade do Estado do Rio de Janeiro- UERJ, Rio de Janeiro, RJ, Brasil); Lúcia Regina Goulart Vilarinho (Universidade Estácio de Sá-UNESA, Rio de Janeiro, RJ, Brasil); Luciana Alaíde Alves Santana (Universidade Federal do Recôncavo 
da Bahia - UFRB, Cruz das Almas, BA, Brasil); Luci dos Santos Bernardi (Universidade Comunitária da Região de Chapecó - UNOCHAPECÓ, Chapecó, SC, Brasil); Luísa Cerdeira (Universidade de Lisboa-ULisboa, Lisboa, Portugal); Luiz Gustavo Lima Freire (Universidade de Lisboa-ULisboa, Lisboa, Portugal);Luzia Queiroz Hippolyto (Instituto Federal de Brasília-IFB, Brasília, DF, Brasil); Marcelo Arancibia Herrera (Universitat Oberta de Catalunya, Catalunya, España); Marcelo Lambach (Universidade Federal do Paraná - UFPR, Curitiba, PR, Brasil); Márcia Aparecida Jacomini (Universidade Federal de São Paulo - Unifesp, São Paulo, SP, Brasil); Márcia Denise Pletsch (Universidade Federal Rural do Rio de Janeiro - UFRRJ, Rio de Janeiro, RJ, Brasil); Márcia Lisbôa Costa de Oliveira (Universidade do Estado do Rio de Janeiro - UERJ, Rio de Janeiro, RJ, Brasil); Margaréte May Berkenbrock Rosito (Universidade Cidade de São Paulo - Unicid, São Paulo, SP, Brasil); Maria Clara Sodré Salgado Gama (Pontifícia Universidade Católica do Rio de Janeiro - PUC-Rio, Rio de Janeiro, RJ, Brasil); Maria Cristina Joly (Universidade de Brasília - UnB, Brasília, DF, Brasil); Maria da Conceição Silva Soares (Universidade do Estado do Rio de Janeiro- UERJ, Rio de Janeiro, RJ, Brasil); Maria da Glória da Veiga Moura (Universidade de Brasília - UnB, Brasília, DF, Brasil); Maria de Fatima da Costa Lippo Acioli (Faculdade de Tecnologia de Alagoas - FAT, Maceió, AL, Brasil); Maria Dilnéia Espíndola Fernandes (Universidade Federal de Mato Grosso do Sul - UFMS, Campo Grande, MS, Brasil); Maria Elisa Rolo Chaleta (Universidade de Évora-UE, Évora, Portugal); Maria Elizete Guimarães Carvalho (Universidade Federal da Paraíba - UFPB, João Pessoa, PB, Brasil); Maria Estela Dal Pai Franco (Universidade Federal do Rio de Janeiro - UFRJ, Rio de Janeiro, RJ, Brasil); Maria Eugénia Ferrão (Universidade da Beira Interior - UBI, Covilhã, Portugal); Maria Isabel Filgueiras Lima Ciasca (Universidade Federal do Ceará - UFCE, Fortaleza, CE, Brasil); Maria João de Carvalho (Universidade de Tras-os-Montes e Alto Douro, Vila Real, Portugal); Maria Vitória Ribas de Oliveira Lima (Universidade de Pernambuco - UPE, Recife, PE, Brasil); Mariângela Spotti Lopes Fujita (Universidade Estadual Paulista - Unesp, São Paulo, SP, Brasil); Marilda Pasqual Schneider (Universidade do Oeste de Santa Catarina - Unoesc, Joaçaba, SC, Brasil); Marilia Andrade Torales Campos (Universidade Federal do Paraná - UFPR, Curitiba, PR, Brasil); Mario Madroñero Morillo (Universidad Nacional de Educación - Unae, Ecuador); Marly de Abreu Costa (Universidade do Estado do Rio de JaneiroUERJ, Rio de Janeiro, RJ, Brasil); Marsyl Bulkool Mettrau (Universidade Salgado de Oliveira - Universo, Niterói, RJ, Brasil); Miguel Pereyra (Universidad de Granada, Granada, España); Mônica Ribeiro da Silva (Universidade Federal do Paraná - UFPR, Curitiba, PR, Brasil); Naura Syria Carapeto Ferreira (Universidade Tuiuti do Paraná - UTP, Curitiba,PR, Brasil); 
Neusa Chaves Batista (Universidade Federal do Rio Grande do Sul - UFRGS, Porto Alegre, RS, Brasil); Nicholas Davies (Universidade Federal Fluminense -UFF, Niterói, RJ, Brasil); Nilva Celestina Carmo (Instituto Federal de Educação, Ciência e Tecnologia do Sudeste de Minas Gerais - IF Sudeste MG, Juiz de Fora. MG, Brasil); Nuno Fraga (Universidade de Madeira, Funchal, Portugal); Rafael Gumiero (Universidade Federal do Sul e Sudeste do Pará - UNIFESSPA, Marabá, PA, Brasil); Olga Moreno-Fernández (Universidade de Sevilha, Sevilha, España); Paulino de Jesus Cardoso (Universidade do Estado de Santa Catarina- UDESC, Florianópolis, SC, Brasil); Paula Guimarães (Universidade de Lisboa - ULISBOA, Lisboa, Portugal); Paulo César Dias (Universidade Católica Portuguesa, Minho, Portugal); Paulo Lima Júnior (Universidade de Brasília - UnB, Brasília, DF, Brasil); Paulo Sérgio Sgarbi Goulart (Universidade do Estado do Rio de Janeiro- UERJ, Rio de Janeiro, RJ, Brasil); Pedro Alberto Barbetta (Universidade Federal de Santa Cantarina - UFSC, Florianópolis, SC, Brasil); Pedro Reis (Universidade de Lisboa -Ulisboa, Lisboa, Portugal); Ramon García Perales (Universidad de Castilla-La Mancha - UCLM, Ciudad Real, España); Ranilce Iosif(Universidade de Alberta, Edmonton, Canadá); Regina Celi Alvarenga de Moura Castro (Universidade Federal do Pará - UFPA, Belém, PA, Brasil); Remi Castioni (Universidade de Brasília - UNB, Brasília, DF, Brasil); Renato de Oliveira Brito (Universidade Católica de Brasília, Brasília, DF, Brasil); Rita de Cássia Prazeres Frangella (Universidade do Estado do Rio de Janeiro- UERJ, Rio de Janeiro, RJ, Brasil); Romualdo Luiz Portela de Oliveira (Universidade de São Paulo - USP, São Paulo, Brasil); Rosana Glat (Universidade do Estado do Rio de Janeiro- UERJ, Rio de Janeiro, RJ, Brasil); Roseli Esquerdo Lopes (Universidade Federal de São Carlos UFSCar, São Carlos, SP, Brasil); Rosilda Arruda Ferreira (Universidade Federal do Recôncavo da Bahia - UFRB - Cruz das Almas, BA, Brasil); Rosilene Beatriz Lopes (Secretaria de Estado da Criança do Distrito Federal, Brasília, DF, Brasil); Sanny Silva da Rosa (Pontifícia Universidade Católica de São Paulo-PUC-SP, São Paulo, SP, Brasil); Sergio Luiz Pereira da Silva (Universidade Federal do Estado do Rio de Janeiro - Unirio, Rio de Janeiro, RJ, Brasil); Silvia Regina dos Santos Coelho (Universidade Católica de Brasília - UCB, Brasília, DF, Brasil); Simone Caputo Gomes (Universidade de São Paulo - USP, São Paulo, SP, Brasil); Sinara Pollom Zardo (Universidade de Brasília - UNB, Brasília, DF, Brasil); Suely da Silva Rodrigues (Fundação Cesgranrio, Rio de Janeiro, RJ, Brasil); Sylvia Garcia Rodrigues (Universidade Federal de Minas Gerais - UFMG, Pampulha, MG, Brasil); Tarso Mazzotti (Universidade Estácio de Sá - Unesa, Rio de Janeiro, RJ, Brasil); Theresa Adrião (Universidade de Campinas - Unicamp, Campinas, SP, Brasil); Terezinha Oliveira (Universidade Estadual de Maringá - UEM, Maringá, PR, 
Brasil); Thiago Alves (Universidade de São Paulo - USP, São Paulo, SP Brasil); Tufi Machado Soares (Universidade Federal de Juiz de Fora - UFJF, Juiz de Fora, MG, Brasil); Vanessa Campos de Lara Jakimiu (Universidade Estadual do Paraná - Unespar - Campus União da Vitória, União da Vitória, PR, Brasil); Vanilda Paiva (Universidade do Estado do Rio de Janeiro- UERJ, Rio de Janeiro, RJ, Brasil); Vera Lucia Felicetti (Universidade La Salle Unilasalle, Canoas, RS, Brasil); Vera Lúcia Jacob Chaves (Universidade Federal do Pará - UFPA, Belém, PA, Brasil); Vera Salvador (Universidade Gama Filho - UGF, Rio de Janeiro, RJ, Brasil); Victor Eduardo Cancino Cancino (Universidad Santo Tomás, Santiago, Chile); Victor Novicki (Universidade Católica de Petrópolis - UCP, Petrópolis, RJ, Brasil); Virgínio Isidro Martins de Sá (Universidade do Minho - U.Minho, Braga, Portugal). 